Vietnam Journal of Mechanics, VAST, Vol.34, No.2 (2012), pp. 123-134

\title{
AN EXPLICIT SECULAR EQUATION OF RAYLEIGH WAVES PROPAGATING ALONG AN OBLIQUELY CUT SURFACE IN A DIRECTIONAL FIBER-REINFORCED COMPOSITE
}

\author{
Pham Chi Vinh ${ }^{1}$, Nguyen Thi Khanh Linh ${ }^{2}$ \\ ${ }^{1}$ Hanoi University of Science, $V N U$ \\ ${ }^{2}$ Water Resources Univetsity of Vietnam
}

\begin{abstract}
The propagation of Rayleigh waves along an obliquely cut surface in a directional fiber-reinforced composite was studied recently by Ohyoshi [Ohyoshi T. Compos Sci Technol 2000; 60; 2191-6]. The author derived the secular equation of the wave, however, it is still implicit. In this paper, a fully explicit secular equation of the wave is obtained by using the method of first integrals. From it we immediately arrive at the secular equation of the wave for the case when the cut surface is parallel to the fiber direction. This secular equation is much more simple than the ones obtained recently by Cerv [Cerv J. Int Rev Mech Eng (IREME) 2008; 2; 762-72] and Cerv et al. [Cerv J et al. Composite Structures 2010; 92; 568-77]. Based on the obtained secular equations some approximate formulas for the velocity of Rayleigh waves are established and it is shown that they are good approximations. The explicit secular equations and the approximate formulas for the velocity derived in this paper are useful for analyzing the effect of the material properties and the orientation of the fiber direction on the Rayleigh wave velocity, especially they are powerful tools for solving the inverse problem: determining the material parameters from the measured values of the velocity.
\end{abstract}

Key words: Fibres, mechanical properties, anisotropy, non-destructive testing, Rayleigh waves.

\section{INTRODUCTION}

The propagation of Rayleigh waves along an obliquely cut surface in a directional fiber-reinforced composite was studied recently by Ohyoshi [1]. The author derived the secular equation of the waves by solving analytically the fully quartic characteristic equation. Among four complex roots of this equation two roots having positive imaginary parts are chosen in order to ensure the decay condition. Since the author could not give the analytical expressions of these two roots, the obtained secular equation is still not explicit. It is therefore not convenient in practical application.

In this paper, we obtain a fully explicit secular equation of the wave by using the method of first integrals $[2,3,4]$. It is a quartic equation in terms of squared velocity. From it we immediately obtain the secular equation for the case when the cut surface is 
parallel to the fiber direction. This secular equation is a cubic equation in terms of squared velocity, and it is much more simple than the ones obtained recently by Cerv [5] and Cerv et al. [6]. Some approximate formulas for the velocity of Rayleigh waves are established for the case when the cut surface is parallel or perpendicular to the fiber direction, and it is shown that they are good approximations. The obtained explicit secular equations and approximate formulas for the velocity will be useful for evaluating the effect of the material properties and the orientation of the fiber direction on the Rayleigh wave velocity, especially they are powerful tools for solving the inverse problem: determining the material parameters from the measured values of the velocity.

\section{RAYLEIGH WAVES ALONG AN OBLIQUELY CUT SURFACE IN A DIRECTIONAL FIBER-REINFORCED COMPOSITE}

\subsection{Basic equations}

Consider a thin fiber-reinforced composite plate occupying the half-space $x_{2} \geq 0$ and its fiber direction is parallel to the $X$-axis (see Fig. 1). Suppose that the $Z$-axis coincides

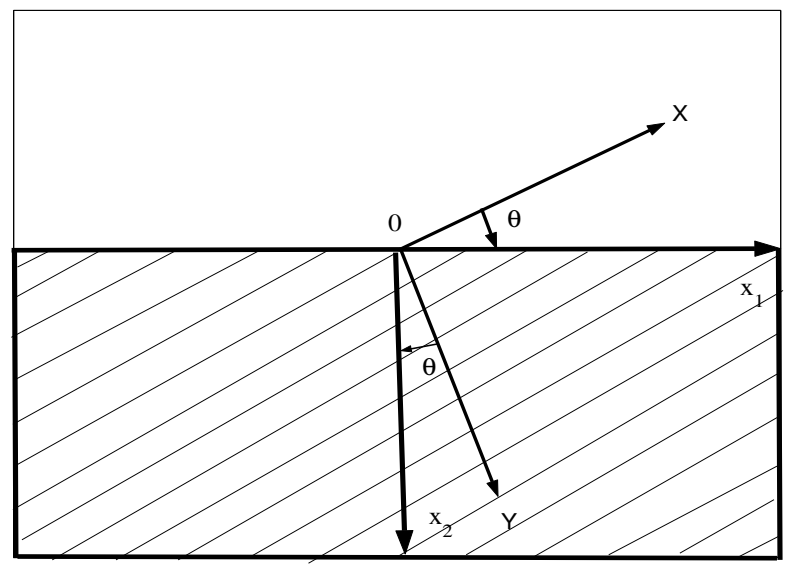

Fig. 1. The thin fiber-reinforced composite plate $x_{2} \geq 0$ whose fiber direction parallel to the $X$-axis, the $Z$-axis coincides with the $x_{3}$-axis and the coordinate system $\left(x_{1}, x_{2}\right)$ is the rotated one from $(X, Y)$ by counter clockwise angle $\theta(0 \leq$ $\theta \leq \pi)$.

with the $x_{3}$-axis and the coordinate system $\left(x_{1}, x_{2}\right)$ is the rotated one from $(X, Y)$ by counter clockwise angle $\theta(0 \leq \theta \leq \pi)$. Suppose that the thin fiber-reinforced composite plate is subjected to the plane stress state

$$
\sigma_{31}=\sigma_{32}=\sigma_{33}=0
$$

Then in the coordinate system $(X, Y)$, the stress-strain relation has the form $[1,6]$

$$
\sigma_{X X}=B_{11} \varepsilon_{X X}+B_{12} \varepsilon_{Y Y}, \sigma_{Y Y}=B_{12} \varepsilon_{X X}+B_{22} \varepsilon_{Y Y}, \sigma_{X Y}=2 B_{66} \varepsilon_{X Y}
$$


where $B_{i j}$ are material (stiffness) coefficients which can be expressed in terms of the engineering constants (Young's and shear moduli, Poisson's ratios) as [1, 6]

$$
B_{11}=\frac{E_{1}}{1-\nu_{12} \nu_{21}}, B_{22}=\frac{E_{2}}{1-\nu_{12} \nu_{21}}, B_{12}=\frac{\nu_{21} E_{1}}{1-\nu_{12} \nu_{21}}=\frac{\nu_{12} E_{2}}{1-\nu_{12} \nu_{21}}, B_{66}=G_{12}
$$

and satisfy the inequalities

$$
B_{k k}>0, k=1,2,6, B_{11} B_{22}-B_{12}^{2}>0
$$

which are necessary and sufficient conditions for the strain energy of the material to be positive define. In the coordinate system $\left(x_{1}, x_{2}\right)$ the stress-strain relation is $[1,6]$

$$
\begin{aligned}
& \sigma_{11}=Q_{11} \varepsilon_{11}+Q_{12} \varepsilon_{22}+2 Q_{16} \varepsilon_{12} \\
& \sigma_{22}=Q_{12} \varepsilon_{11}+Q_{22} \varepsilon_{22}+2 Q_{26} \varepsilon_{12} \\
& \sigma_{12}=Q_{16} \varepsilon_{11}+Q_{26} \varepsilon_{22}+2 Q_{66} \varepsilon_{12}
\end{aligned}
$$

where [1]

$$
\begin{aligned}
& Q_{11}=B_{11} c_{\theta}^{4}+2\left(B_{12}+2 B_{66}\right) c_{\theta}^{2} s_{\theta}^{2}+B_{22} s_{\theta}^{4} \\
& Q_{22}=B_{11} s_{\theta}^{4}+2\left(B_{12}+2 B_{66}\right) c_{\theta}^{2} s_{\theta}^{2}+B_{22} c_{\theta}^{4} \\
& Q_{12}=\left(B_{11}+B_{22}-4 B_{66}\right) c_{\theta}^{2} s_{\theta}^{2}+B_{12}\left(c_{\theta}^{4}+s_{\theta}^{4}\right) \\
& Q_{66}=\left(B_{11}+B_{22}-2 B_{12}-2 B_{66}\right) c_{\theta}^{2} s_{\theta}^{2}+B_{66}\left(c_{\theta}^{4}+s_{\theta}^{4}\right) \\
& Q_{16}=-\left(B_{11}-B_{12}-2 B_{66}\right) c_{\theta}^{3} s_{\theta}-\left(B_{12}-B_{22}+2 B_{66}\right) c_{\theta} s_{\theta}^{3} \\
& Q_{26}=-\left(B_{11}-B_{12}-2 B_{66}\right) c_{\theta} s_{\theta}^{3}-\left(B_{12}-B_{22}+2 B_{66}\right) c_{\theta}^{3} s_{\theta}
\end{aligned}
$$

in which $c_{\theta}:=\cos \theta, s_{\theta}:=\sin \theta$ and the strain $\varepsilon_{i j}$ are expressed in terms of the displacement gradients $u_{m, n}$ as

$$
\varepsilon_{11}=u_{1,1}, \quad \varepsilon_{22}=u_{2,2}, \quad \varepsilon_{12}=\left(u_{1,2}+u_{2,1}\right) / 2
$$

On view of (6) it is easily to show that

$$
\begin{aligned}
Q_{k k}(\theta)= & Q_{k k}(\pi-\theta)(k=1,2,6), Q_{12}(\theta)=Q_{12}(\pi-\theta) \\
& Q_{k 6}(\theta)=-Q_{k 6}(\pi-\theta)(k=1,2)
\end{aligned}
$$

and if $B_{11}=B_{22}\left(\Leftrightarrow E_{1}=E_{2}\right)$ :

$$
\begin{aligned}
Q_{k k}(\theta)= & Q_{k k}(\pi / 2-\theta)(k=1,2,6), Q_{12}(\theta)=Q_{12}(\pi / 2-\theta) \\
& Q_{k 6}(\theta)=-Q_{k 6}(\pi / 2-\theta)(k=1,2)
\end{aligned}
$$

In the absence of body forces, equations of motion are [1]

$$
\sigma_{11,1}+\sigma_{12,2}=\rho \ddot{u_{1}}, \sigma_{12,1}+\sigma_{22,2}=\rho \ddot{u_{2}}
$$

Following the same procedure carried out in [7] Section 2, from Eqs. (5), (7) and (10) we have

$$
\left[\begin{array}{l}
u^{\prime} \\
\sigma^{\prime}
\end{array}\right]=N\left[\begin{array}{l}
u \\
\sigma
\end{array}\right]
$$


where $u=\left[u_{1}, u_{2}\right]^{T}, \sigma=\left[\sigma_{12}, \sigma_{22}\right]^{T}$, the symbol $T$ indicates the transpose of matrices, the prime indicates the derivative with respect to $x_{2}$ and

$$
\begin{aligned}
& N=\left[\begin{array}{cc}
N_{1} & N_{2} \\
K & N_{3}
\end{array}\right], \quad N_{1}=\left[\begin{array}{cc}
\left(d_{1} / d\right) \partial_{1} & -\partial_{1} \\
-\left(d_{2} / d\right) \partial_{1} & 0
\end{array}\right], \quad N_{2}=\frac{1}{d}\left[\begin{array}{cc}
Q_{22} & -Q_{26} \\
-Q_{26} & Q_{66}
\end{array}\right] \\
& K=\left[\begin{array}{cc}
\rho \partial_{t}^{2}-\left(d_{3} / d\right) \partial_{1}^{2} & 0 \\
0 & \rho \partial_{t}^{2}
\end{array}\right], \quad N_{3}=N_{1}^{T}
\end{aligned}
$$

Here we use the notations: $\partial_{1}=\partial /\left(\partial x_{1}\right), \partial_{1}^{2}=\partial^{2} /\left(\partial x_{1}^{2}\right), \partial_{t}^{2}=\partial^{2} /\left(\partial t^{2}\right)$ and

$$
\begin{gathered}
d=Q_{22} Q_{66}-Q_{26}^{2}, d_{1}=Q_{12} Q_{26}-Q_{22} Q_{16} \\
d_{2}=Q_{12} Q_{66}-Q_{16} Q_{26}, d_{3}=Q_{11} d+Q_{16} d_{1}-Q_{12} d_{2}
\end{gathered}
$$

From (8), (9) and (13) it implies that

$$
d(\theta)=d(\pi-\theta), d_{1}(\theta)=-d_{1}(\pi-\theta), d_{k}(\theta)=d_{k}(\pi-\theta)(k=2,3)
$$

and if $B_{11}=B_{22}\left(\Leftrightarrow E_{1}=E_{2}\right)$

$$
d(\theta)=d(\pi / 2-\theta), d_{1}(\theta)=-d_{1}(\pi / 2-\theta), d_{k}(\theta)=d_{k}(\pi / 2-\theta)(k=2,3)
$$

In addition to Eq. (11), the displacement vector $u$ and the traction vector $\sigma$ are required to satisfy the decay condition at the infinity

$$
u(+\infty)=0, \sigma(+\infty)=0
$$

and the free-traction condition at the edge $x_{2}=0$

$$
\sigma(0)=0
$$

\subsection{Explicit secular equation}

Now we consider the propagation of a Rayleigh wave, travelling with velocity $c$ and wave number $k$ in the $x_{1}$-direction. The components $u_{1}, u_{2}$ of the displacement vector and $\sigma_{12}, \sigma_{22}$ of the traction vector at the planes $x_{3}=$ const are found in the form

$$
\left\{u_{1}, u_{2}, \sigma_{12}, \sigma_{22}\right\}\left(x_{1}, x_{2}, t\right)=\left\{U_{1}\left(k x_{2}\right), U_{2}\left(k x_{2}\right), i k V_{1}\left(k x_{2}\right), i k V_{2}\left(k x_{2}\right)\right\} \mathrm{e}^{i k\left(x_{1}-c t\right)}
$$

Substituting (18) into (11) yields

$$
\left[\begin{array}{l}
U^{\prime} \\
V^{\prime}
\end{array}\right]=i M\left[\begin{array}{l}
U \\
V
\end{array}\right]
$$

where $U=\left[U_{1} U_{2}\right]^{T}, V=\left[V_{1} V_{2}\right]^{T}$, and

$$
\begin{array}{cc}
M=\left[\begin{array}{cc}
M_{1} & M_{2} \\
Q & M_{3}
\end{array}\right], & M_{1}=\left[\begin{array}{cc}
d_{1} / d & -1 \\
-d_{2} / d & 0
\end{array}\right], \\
Q=\left[\begin{array}{cc}
X-d_{3} / d & 0 \\
0 & X
\end{array}\right], & M_{2}=\frac{1}{d}\left[\begin{array}{cc}
Q_{22} & -Q_{26} \\
-Q_{26} & Q_{66}
\end{array}\right], \\
& M_{1}^{T}
\end{array}
$$

$X=\rho c^{2}$, the prime in Eq. (19) indicates the derivative with respect to $y=k x_{2}$. From (20), one can see that the characteristic equation $|M-p I|=0$ of Eq. (19) is a fully quartic equation for $p$ (see also Eq. (18) in Ref. [1]), therefore we should employ the method of first integrals $[2,3]$ in order to obtain the explicit secular equation of the wave. 
Eliminating $U$ from (19), we have

$$
\alpha V^{\prime \prime}-i \beta V^{\prime}-\gamma V=0
$$

where the matrices $\alpha, \beta, \gamma$ are given by

$$
\begin{gathered}
\alpha=Q^{-1}=\left[\begin{array}{cc}
\frac{d}{d X-d_{3}} & 0 \\
0 & \frac{1}{X}
\end{array}\right] \\
\beta=M_{1} Q^{-1}+Q^{-1} M_{3}=\left[\begin{array}{cc}
\frac{2 d_{1}}{d X-d_{3}} & -\frac{1}{X}-\frac{d_{2}}{d X-d_{3}} \\
-\frac{1}{X}-\frac{d_{2}}{d X-d_{3}} & 0
\end{array}\right] \\
\gamma=M_{1} Q^{-1} M_{3}-M_{2}=\left[\begin{array}{cc}
\frac{d_{1}^{2}}{d\left(d X-d_{3}\right)}+\frac{1}{X}-\frac{Q_{22}}{d} & -\frac{d_{1} d_{2}}{d\left(d X-d_{3}\right)}+\frac{Q_{26}}{d} \\
-\frac{d_{1} d_{2}}{d\left(d X-d_{3}\right)}+\frac{Q_{26}^{2}}{d} & \frac{d_{66}^{2}}{d\left(d X-d_{3}\right)}-\frac{Q_{66}}{d}
\end{array}\right]
\end{gathered}
$$

Note that $\alpha, \beta, \gamma$ are symmetric real matrices. From (16)-(18) it follows

$$
V(0)=V(+\infty)=0
$$

Our task now is to solve Eq. (21) along with the boundary condition (25). In the component form Eq. (21) is written as follows

$$
\alpha_{k l} V_{l}^{\prime \prime}-i \beta_{k l} V_{l}^{\prime}-\gamma_{k l} V_{l}=0, \quad(k, l=1,2)
$$

Multiplying two sides of Eq. (26) by $i \bar{\Sigma}_{m}$ and then adding the resulting equation to its conjugation give

$$
\alpha_{k l}\left(i V_{l}^{\prime \prime} \bar{V}_{m}+\overline{i V_{l}^{\prime \prime}} V_{m}\right)+\beta_{k l}\left(V_{l}^{\prime} \bar{V}_{m}+\overline{V_{l}^{\prime}} V_{m}\right)+\gamma_{k l}\left(V_{l} \overline{i V}_{m}+\bar{V}_{l} i V_{m}\right)=0
$$

where the bar indicates the complex conjugation. Now we introduce $2 \times 2$-matrices $D, E, F$ whose elements are defined as follows

$$
D_{l m}=<i V_{l}^{\prime \prime}, V_{m}>, \quad E_{l m}=<V_{l}^{\prime}, V_{m}>, \quad F_{l m}=<V_{l}, i V_{m}>, \quad l, m=1,2
$$

where: $\langle\varphi, g\rangle=\int_{0}^{+\infty}(\varphi \bar{g}+\bar{\varphi} g) d y$. From (25) and (28), we find out that $D, E, F$ being antisymmetric, i. e.

$$
D=\left[\begin{array}{cc}
0 & d \\
-d & 0
\end{array}\right], \quad E=\left[\begin{array}{cc}
0 & e \\
-e & 0
\end{array}\right], \quad F=\left[\begin{array}{cc}
0 & f \\
-f & 0
\end{array}\right]
$$

Now, integrating Eq. (27) from 0 to $+\infty$ provides

$$
\alpha D+\beta E+\gamma F=0
$$


From (29) and (30) it follows

$$
\left\{\begin{array}{l}
\alpha_{11} d+\beta_{11} e+\gamma_{11} f=0 \\
\alpha_{12} d+\beta_{12} e+\gamma_{12} f=0 \\
\alpha_{22} d+\beta_{22} e+\gamma_{22} f=0
\end{array}\right.
$$

Thus, we have

$$
\left|\begin{array}{lll}
\alpha_{11} & \beta_{11} & \gamma_{11} \\
\alpha_{12} & \beta_{12} & \gamma_{12} \\
\alpha_{22} & \beta_{22} & \gamma_{22}
\end{array}\right|=0
$$

in order that the homogeneous linear system (31) has a non trivial solution. Introducing (22)-(24) into (32) yields

$$
\begin{aligned}
& F(X, \theta) \equiv d X^{2}\left[\left(d+d_{2}\right) X-d_{3}\right]\left[d_{2}^{2}-Q_{66}\left(d X-d_{3}\right)\right]+ \\
&+\left(d X-d_{3}\right)\left[\left(d+d_{2}\right) X-d_{3}\right]\left[Q_{22} d X^{2}-\left(d^{2}+d_{1}^{2}+Q_{22} d_{3}\right) X+d d_{3}\right] \\
&-2 d_{1} X^{2}\left(d X-d_{3}\right)\left[Q_{26}\left(d X-d_{3}\right)-d_{1} d_{2}\right]=0
\end{aligned}
$$

Equation (33) is the desired explicit secular equation and it is a quartic equation for $X=\rho c^{2}$. On use of (8), (9), (14) and (15) one can prove that $F(X, \theta)=F(X, \pi-\theta)$ and if $B_{11}=B_{22}\left(\Leftrightarrow E_{1}=E_{2}\right)$, then $F(X, \theta)=F(X, \pi / 2-\theta)$. From these facts and Eq. (33) it follows

$$
X(\theta)=X(\pi-\theta) \text { and if } B_{11}=B_{22} \text { then } X(\theta)=X(\pi / 2-\theta)
$$

or equivalently

$$
c_{R}(\theta)=c_{R}(\pi-\theta) \text { and if } B_{11}=B_{22} \text { then } c_{R}(\theta)=c_{R}(\pi / 2-\theta)
$$

where $c_{R}$ is the velocity of Rayleigh waves.

\section{Remark 1:}

The first of (35) says that in the interval $[0, \pi]$ the curve $c_{R}=c_{R}(\theta)$ has always one symmetry axis, namely the line $\theta=\pi / 2$. If $B_{11}=B_{22}\left(\Leftrightarrow E_{1}=E_{2}\right)$, then in addition, the lines $\theta=\pi / 4$ and $\theta=3 \pi / 4$ are symmetry axes of the curve $c_{R}=c_{R}(\theta)$ in the interval $[0, \pi / 2]$ and $[\pi / 2, \pi]$, respectively, by the second of (35). With this fact now we can understand why the curves $c_{R}=c_{R}(\theta)$ in the figures 14, 15, 18, 19 in [6] have the symmetry axis $\theta=\pi / 2$ in the interval $[0, \pi]$, while the curves $c_{R}=c_{R}(\theta)$ in the figures 16 , $17,20,21$ in [6] have the symmetry axis $\theta=\pi / 4$ in the interval $[0, \pi / 2]$ and the symmetry axis $\theta=3 \pi / 4$ in the interval $[\pi / 2, \pi]$, in addition.

By dividing two sides of Eq. $(33)$ by $\left(B_{66}\right)^{11}$ we obtain the dimensionless secular equation, namely

$$
\begin{aligned}
f\left(x, r_{1}, r_{2}, r_{3}, \theta\right) & \equiv \hat{d} x^{2}\left[\left(\hat{d}+\hat{d}_{2}\right) x-\hat{d}_{3}\right]\left[\hat{d}_{2}^{2}-\hat{Q}_{66}\left(\hat{d} x-\hat{d}_{3}\right)\right] \\
& +\left(\hat{d} x-\hat{d}_{3}\right)\left[\left(\hat{d}+\hat{d}_{2}\right) x-\hat{d}_{3}\right]\left[\hat{Q}_{22} \hat{d} x^{2}-\left(\hat{d}^{2}+\hat{d}_{1}^{2}+\hat{Q}_{22} \hat{d}_{3}\right) x+\hat{d} \hat{d}_{3}\right] \\
& -2 \hat{d}_{1} x^{2}\left(\hat{d} x-\hat{d}_{3}\right)\left[\hat{Q}_{26}\left(\hat{d} x-\hat{d}_{3}\right)-\hat{d}_{1} \hat{d}_{2}\right]=0
\end{aligned}
$$


where $x=X / B_{66}$ called the squared dimensionless velocity, $r_{1}=B_{11} / B_{66}, r_{2}=B_{22} / B_{66}$, $r_{3}=B_{12} / B_{66}$, and

$$
\begin{aligned}
& \hat{Q}_{11}=r_{1} c_{\theta}^{4}+2\left(r_{3}+2\right) c_{\theta}^{2} s_{\theta}^{2}+r_{2} s_{\theta}^{4}, \hat{Q}_{22}=r_{1} s_{\theta}^{4}+2\left(r_{3}+2\right) c_{\theta}^{2} s_{\theta}^{2}+r_{2} c_{\theta}^{4} \\
& \hat{Q}_{12}=\left(r_{1}+r_{2}-4\right) c_{\theta}^{2} s_{\theta}^{2}+r_{3}\left(c_{\theta}^{4}+s_{\theta}^{4}\right), \hat{Q}_{66}=\left(r_{1}+r_{2}-2 r_{3}-2\right) c_{\theta}^{2} s_{\theta}^{2}+c_{\theta}^{4}+s_{\theta}^{4} \\
& \hat{Q}_{16}=-\left(r_{1}-r_{3}-2\right) c_{\theta}^{3} s_{\theta}-\left(r_{3}-r_{2}+2\right) c_{\theta} s_{\theta}^{3} \\
& \hat{Q}_{26}=-\left(r_{1}-r_{3}-2\right) c_{\theta} s_{\theta}^{3}-\left(r_{3}-r_{2}+2\right) c_{\theta}^{3} s_{\theta} \\
& \hat{d}=\hat{Q}_{22} \hat{Q}_{66}-\hat{Q}_{26}^{2}, \hat{d}_{1}=\hat{Q}_{12} \hat{Q}_{26}-\hat{Q}_{22} \hat{Q}_{16} \\
& \hat{d}_{2}=\hat{Q}_{12} \hat{Q}_{66}-\hat{Q}_{16} \hat{Q}_{26}, \hat{d}_{3}=\hat{Q}_{11} \hat{d}+\hat{Q}_{16} \hat{d}_{1}-\hat{Q}_{12} \hat{d}_{2}
\end{aligned}
$$

It is clear from (36) that $x$ depends on three dimensionless material parameters $r_{k}(k=1,2,3)$ and the angle $\theta$ (the orientation of the fiber direction), and from (35) it follows

$$
x\left(r_{1}, r_{2}, r_{3}, \theta\right)=x\left(r_{1}, r_{2}, r_{3}, \pi-\theta\right)
$$

and if $B_{11}=B_{22}\left(\Leftrightarrow E_{1}=E_{2}\right)$, we have in addition

$$
x\left(r_{1}, r_{2}, r_{3}, \theta\right)=x\left(r_{1}, r_{2}, r_{3}, \pi / 2-\theta\right)
$$

On view of (4) the dimensionless $r_{k}$ are subjected to: $r_{1}>0, r_{2}>0$ and $r_{3}^{2}<r_{1} r_{2}$. The explicit secular equations (33) and (36) are useful for evaluating the effect of the orientation of the fiber direction and of the material properties on the velocity of Rayleigh waves. They are also convenient tools for solving the inverse problem: determining the material parameters from measured values of the velocity.

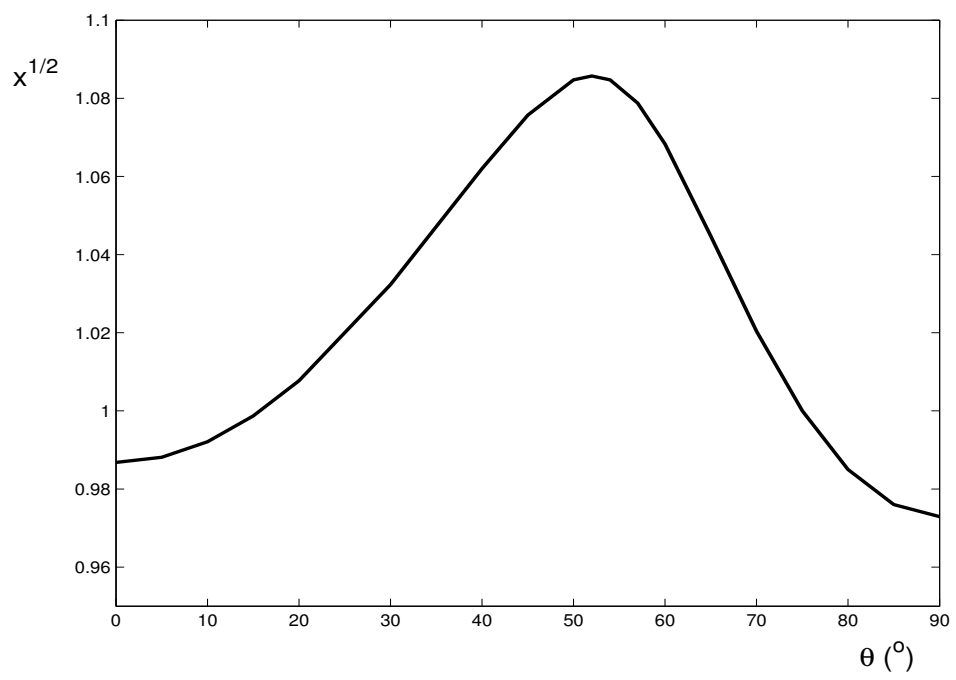

Fig. 2. Dependence of $\sqrt{x}=c_{R} / \sqrt{B_{66} / \rho}$ on the orientation $\theta$ of the fiber direction for the material SE84LV: $E_{1}=114.20 \mathrm{GPa}, E_{2}=8.80 \mathrm{GPa}, G_{12}=5.15 \mathrm{GPa}$, $\nu_{12}=0.28, \rho=1540 \mathrm{~kg} \mathrm{~m}^{-3}$. 
As examples, we apply the secular equation (36) to draw the curves $x=x(\theta)$ for the materials: SE84LV and Fibredux whose material constants are listed in Table 1 in Ref. [6]. These curves are presented in Figs. 2, 3. By (38) it is sufficient to draw these curves in the interval $\left[0,90^{\circ}\right]$.

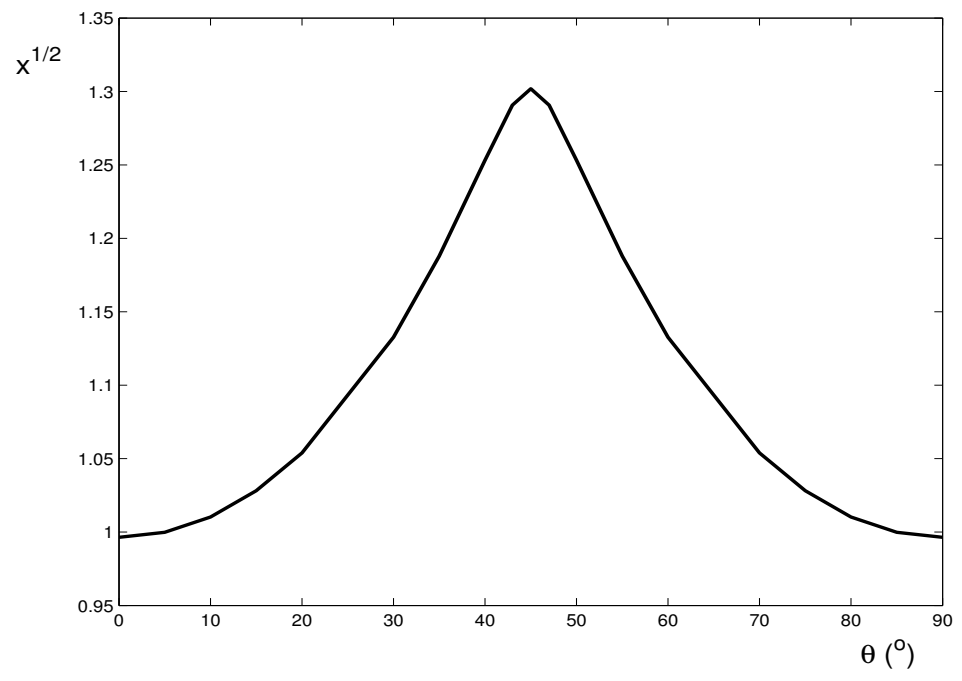

Fig. 3. Dependence of $\sqrt{x}=c_{R} / \sqrt{B_{66} / \rho}$ on the orientation $\theta$ of the fiber direction for the material Fibredux: $E_{1}=E_{2}=62.2 \mathrm{GPa}, G_{12}=5.08 \mathrm{GPa}, \nu_{12}=0.041$, $\rho=1560 \mathrm{~kg} \mathrm{~m}^{-3}$.

\subsection{Special cases}

When $\theta=0$, from (6) and (13) we have

$$
\begin{aligned}
& Q_{k k}=B_{k k}, k=1,2,6, Q_{12}=B_{12}, Q_{16}=Q_{26}=0 \\
& d=B_{22} B_{66}, d_{1}=0, d_{2}=B_{12} B_{66}, d_{3}=B_{66}\left(B_{11} B_{22}-B_{12}^{2}\right)
\end{aligned}
$$

Introducing (40) into (33) yields a cubic equation for $X$, namely

$$
B_{22} B_{66} X^{2}\left(B_{11}-X\right)-\left(B_{66}-X\right)\left[B_{12}^{2}-B_{22}\left(B_{11}-X\right)\right]^{2}=0
$$

This is the secular equation of Rayleigh waves for the case when the fiber direction is parallel to the edge of the composite plate. One can see that if a Rayleigh wave exists then

$$
0<X<\min \left\{B_{11}, B_{66}\right\}
$$

\section{Remark 2:}

The secular equation (41) is much more simple than the secular equations (13), (21) in [5] obtained recently by Cerv, and it is valid for any orthotropic elastic materials.

When the material is isotropic: $B_{11}=B_{22}=\lambda+2 \mu, B_{12}=\lambda, B_{66}=\mu, \lambda, \mu$ are the usual Lame constants, Eq. (41) reduces to the well-known Rayleigh equation

$$
x^{3}-8 x^{2}+8(3-2 \delta) x-16(1-\delta)=0
$$

where $x=\rho c^{2} / \mu, \delta=\mu /(\lambda+2 \mu)$. 
When $\theta=\pi / 2$, by (6) and (13) we have:

$Q_{11}=B_{22}, Q_{22}=B_{11}, Q_{66}=B_{66}, Q_{12}=B_{12}, Q_{12}=B_{12}, Q_{16}=Q_{26}=0$

$d=B_{11} B_{66}, d_{1}=0, d_{2}=B_{12} B_{66}, d_{3}=B_{66}\left(B_{11} B_{22}-B_{12}^{2}\right)$

Using (44) into (33) we have:

$$
B_{11} B_{66} X^{2}\left(B_{22}-X\right)-\left(B_{66}-X\right)\left[B_{12}^{2}-B_{11}\left(B_{22}-X\right)\right]^{2}=0
$$

in which $0<X<\min \left\{B_{22}, B_{66}\right\}$. Equation (45) is the secular equation of Rayleigh waves for the case when the fiber direction is perpendicular to the edge of the composite plate. It is a also cubic equation for $X$.

\section{APPROXIMATE FORMULAS FOR THE RAYLEIGH WAVE VELOCITY}

In this section we establish some approximate formulas for the Rayleigh wave velocity for the cases when the fiber direction is parallel or perpendicular to the edge of the composite.

After expanding and rearranging Eq. (41) becomes:

$$
\begin{aligned}
& B_{22}\left(B_{22}-B_{66}\right) X^{3}+B_{22}\left(B_{11} B_{66}-B_{22} B_{66}-2 \delta_{12}\right) X^{2} \\
& +\delta_{12}\left(\delta_{12}+2 B_{22} B_{66}\right) X-B_{22} \delta_{12}^{2}=0
\end{aligned}
$$

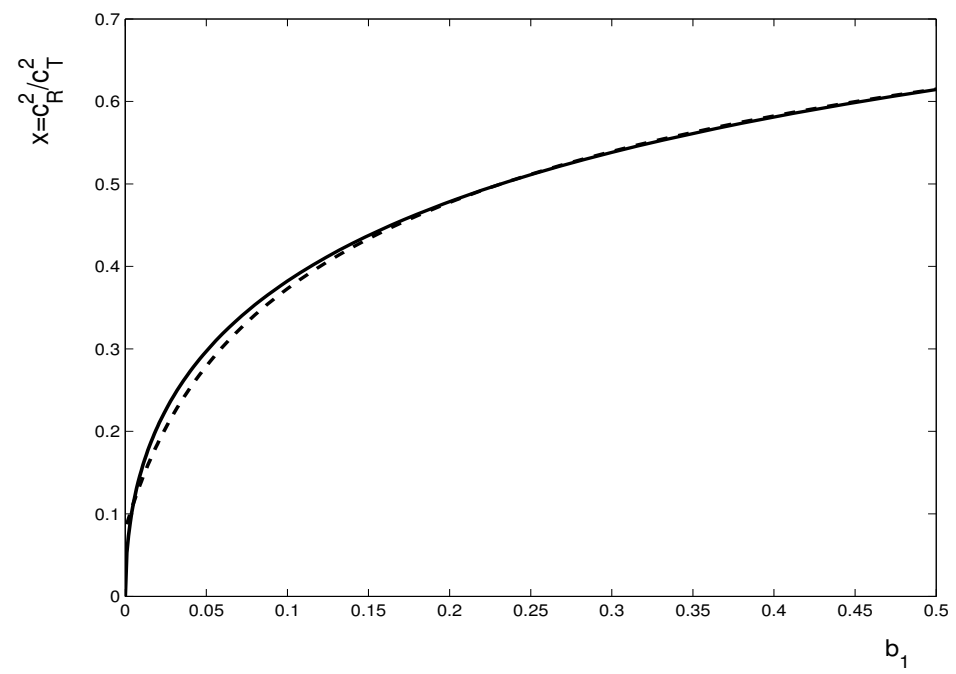

Fig. 4. Dependence on $b_{1} \in[0,0.5]$ of $x=c_{R}^{2} / c_{T}^{2}$ that is calculated by solving directly the secular equation (41) (solid line), by the approximate formula (51)(52) (dashed line). Here we take $b_{2}=0.9937, b_{3}=1.7532$.

Dividing two sides of Eq. (46) by $\left(B_{66}\right)^{5}(>0)$ leads to

$$
m_{3} x^{3}+m_{2} x^{2}+m_{1} x+m_{0}=0,0<x=X / B_{66}<1
$$


where

$$
\begin{aligned}
& m_{3}=b_{1} b_{3}\left(1-b_{1} b_{3}\right), m_{2}=b_{1} b_{3}^{2}\left(1-b_{1}-2 b_{1} b_{2} b_{3}\right) \\
& m_{1}=b_{1}^{2} b_{2} b_{3}^{3}\left(b_{2} b_{3}+3\right), m_{0}=-b_{1}^{2} b_{2}^{2} b_{3}^{4} \\
& b_{1}=B_{22} / B_{11}, b_{2}=1-B_{12}^{2} /\left(B_{11} B_{22}\right), b_{3}=B_{11} / B_{66}
\end{aligned}
$$

According to Vinh and Malischewsky [8], in the interval [0, 1], the best approximate second-order polynomial of $x^{3}$ in the sense of least squares is

$$
1.5 x^{2}-0.6 x+0.05
$$

Introducing (49) into (47) yields a quadratic equation, namely

$$
\left(m_{2}+1.5 m_{3}\right) x^{2}-\left(0.6 m_{3}-m_{1}\right) x+m_{0}+0.05 m_{3}=0
$$

whose solution corresponding to the Rayleigh-edge wave is

$$
x=\frac{B-\sqrt{B^{2}-4 A C}}{2 A}
$$

where

$$
\begin{aligned}
& A=b_{1} b_{3}\left[b_{3}\left(1+0.5 b_{1}-2 b_{1} b_{2} b_{3}\right)-1.5\right] \\
& B=b_{1} b_{3}\left[0.6\left(b_{1} b_{3}-1\right)-b_{1} b_{2} b_{3}^{2}\left(b_{2} b_{3}+2\right)\right] \\
& C=0.05 b_{1} b_{3}\left(b_{1} b_{3}-1\right)-b_{1}^{2} b_{2}^{2} b_{3}^{4}
\end{aligned}
$$

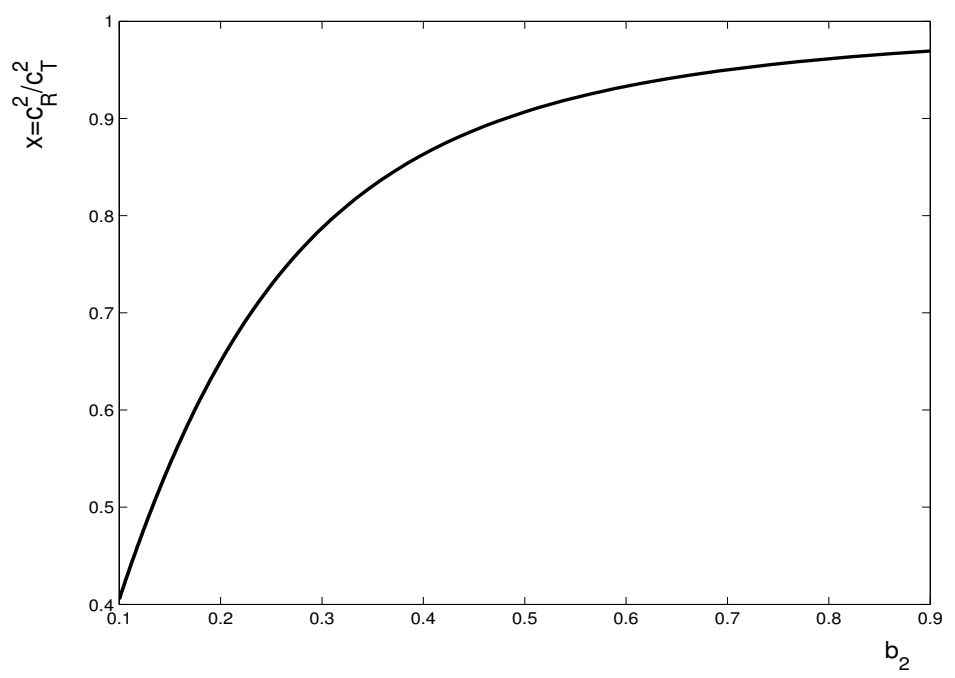

Fig. 5. Dependence on $b_{2} \in[0.1,0.9]$ of $x=c_{R}^{2} / c_{T}^{2}$ that is calculated by solving directly the secular equation (41), by the approximate formulas (51)-(52) and (51)-(53). They almost totally coincide with each other. Here we take $b_{1}=0.077$, $b_{3}=22.7813$. 
If we use the best approximate second-order polynomial of $x^{3}$ in the space $C[0,1]$, namely: $1.5 x^{2}-0.5625 x+0.03125$ (see [8]), then $x$ is given by (51) in which

$$
\begin{aligned}
& A=b_{1} b_{3}\left[b_{3}\left(1+0.5 b_{1}-2 b_{1} b_{2} b_{3}\right)-1.5\right] \\
& B=b_{1} b_{3}\left[0.5625\left(b_{1} b_{3}-1\right)-b_{1} b_{2} b_{3}^{2}\left(b_{2} b_{3}+2\right)\right] \\
& C=0.03125 b_{1} b_{3}\left(b_{1} b_{3}-1\right)-b_{1}^{2} b_{2}^{2} b_{3}^{4} .
\end{aligned}
$$

The Figs. 4, 5,6 show the dependence of the squared dimensionless velocity $x$ on the

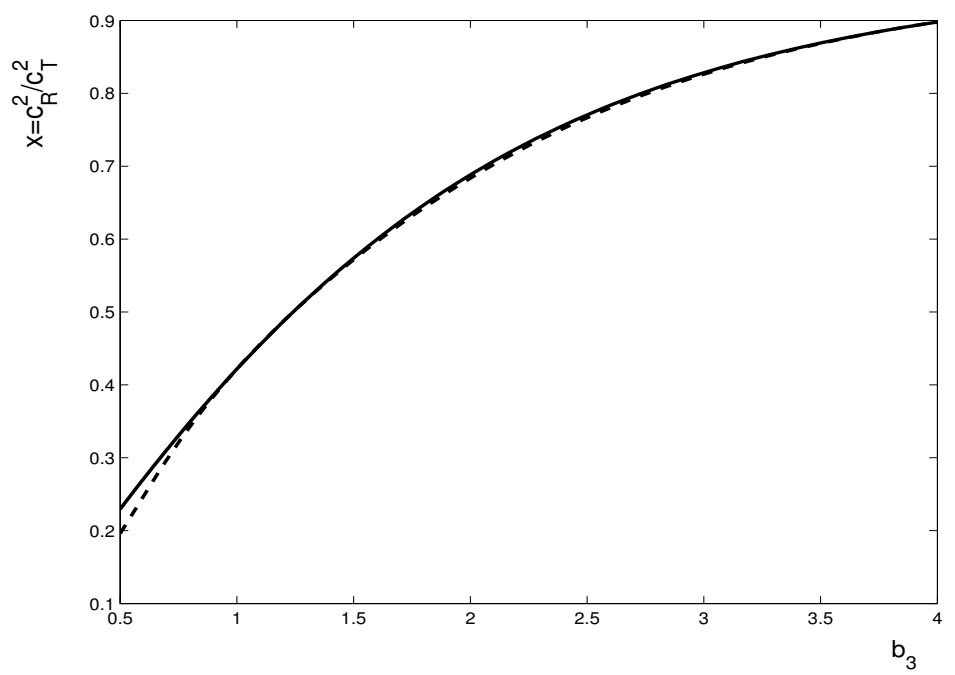

Fig. 6. Dependence on $b_{3} \in[0.5,4]$ of $x=c_{R}^{2} / c_{T}^{2}$ that is calculated by solving directly the secular equation (41) (solid line), by the approximate formula (51)(52) (dashed line). Here we take $b_{1}=1, b_{2}=0.8432$.

dimensionless material parameters $b_{k}$. It is shown from these figures that the formulas (51)(52) and (51)-(53) are highly accurate approximations. They are thrtefore significant in practical applications, especially for solving the inverse problem: determining the material parameters of the composite from measured values of the velocity of Rayleigh waves. Since these formulas are good approximations as shown from the Figs. 4, 5, 6, they are useful in practical applications.

\section{Remark 3:}

When the fiber direction is perpendicular to the edge of the composite, the Rayleigh wave velocity is approximately calculated by (51)-(52) or (51)-(53) in which $b_{1}=B_{11} / B_{22}$, $b_{2}=1-B_{12}^{2} /\left(B_{11} B_{22}\right), b_{3}=B_{22} / B_{66}$.

\section{CONCLUSIONS}

In this paper, a fully explicit secular equation of Rayleigh waves along an obliquely cut surface in a directional fiber-reinforced composite has been derived by using the method of first integrals. Based on it, the symmetry properties of the dependence of the Rayleigh wave velocity on the orientation of the fiber direction, which were indicated in Refs. $[5,6]$ 
throught numerical studies, are proved. From the obtained explicit secular equation we immediately derive the secular equation of the wave for the case when the cut surface is parallel to the fiber direction, and it is much more simple than the ones obtained recently by Cerv [5] and Cerv et al. [6]. Some approximate formulas for the Rayleigh wave speed for the cases when the fiber direction is parallel or perpendicular to the edge of the composite plate are established. It is shown that they are good approximations. The obtained explicit secular equation and approximate formulas for the velocity would be useful in practical applications.

\section{ACKNOWLEDGMENTS}

The work was supported by the Vietnam National Foundation For Science and Technology Development (NAFOSTED) under Grant No. 107.02-2010.07.

\section{REFERENCES}

[1] Ohyoshi T., The propagation of Rayleigh waves along an obliquely cut surface in a directional fibre-reinforced composite, Compos Sci Technol, 60, (2000), 2191 - 2196.

[2] Mozhaev V G., Some new ideas in the theory of surface acoustic waves in anisotropic media, IUTAM Symposium on Anisotropy, Inhomogeneity and Nonlinearity in Solid Mechanics, edited by Parker D F and England A H, Kluwer Acad Publ, (1995), 455 - 462.

[3] Destrade M., The explicit secular equation for surface acoustic waves in monoclinic elastic crystals, J. Acoust Soc. Am., 109, (2001), 1398 - 1402.

[4] Pham Chi Vinh, Trinh Thi Thanh Hue, Dinh Van Quang, Nguyen Thi Khanh Linh and Nguyen Thi Nam, Method of first integrals and interface and surface waves, Vietnam Journal of Mechanics, VAST, 32(2) (2010), 107 - 120.

[5] Cerv J., The Influence of Material Properties on the Behaviour of Rayleigh Edge Waves in Thin Orthotropic Media, Int. Rev. Mech. Eng. (IREME), 2, (2008), 762 - 772.

[6] Cerv J., Kroupa T., Trnka J., Influence of principal material directions of thin orthotropic structures on Rayleigh-edge wave velocity, Composite Structures, 92, (2010), 568 - 577.

[7] Pham Chi Vinh, Explicit secular equations of Rayleigh waves in elastic media under the influence of gravity and initial stress, Appl. Math. Comput., 215, (2009), 395 - 404.

[8] Pham Chi Vinh, Malischewsky P., An approach for obtaining approximate formulas for the Rayleigh wave velocity, Wave Motion, 44, (2007), 549 - 562.

Received November 14, 2011 